\title{
O Teatro e a educação em saúde na escola: Relato de Experiência
}

\author{
Theater and health education in school: Experience Report
}

\author{
Eduardo Alexander Júlio César Fonseca Lucas ${ }^{1}$ Lucas Lima de Carvalho² Lucas Rodrigues Claro ${ }^{3}$ Amanda \\ dos Santos Cabral ${ }^{4}$ Marcela Pereira da Silva Mello ${ }^{5}$ Regina Izabella Mendes da Costa ${ }^{6}$ Maria Cristina Dias da \\ Silva ${ }^{7}$ Bruna Liane Passos Lucas ${ }^{8}$ Antonio Eduardo Vieira dos Santos ${ }^{9}$ Ravini dos Santos Fernandes Vieira \\ dos Santos ${ }^{10}$ Alexandre Oliveira Telles ${ }^{11}$ Vera Lucia Rabello de Castro Halfoun ${ }^{12}$ Maria Kátia Gomes ${ }^{13}$
}

1 Professor. Universidade Federal do Rio de Janeiro (UFRJ), Brasil. E-mail: eduardoalexander@gmail.com

2 Graduando. Universidade Federal do Rio de Janeiro (UFRJ), Brasil. E-mail: lucaslimac17@gmail.com

3 Graduando. Universidade Federal do Rio de Janeiro (UFRJ), Brasil. E-mail: lucasclaro222@gmail.com

4 Graduanda. Universidade Federal do Rio de Janeiro (UFRJ), Brasil. E-mail: amandascabral1@gmail.com

5 Graduanda. Universidade Federal do Rio de Janeiro (UFRJ), Brasil. E-mail: marcela-mello@hotmail.com

6 Graduanda. Universidade Federal do Rio de Janeiro (UFRJ), Brasil. E-mail: izahmdc@gmail.com

7 Enfermeira. Universidade Federal do Rio de Janeiro (UFRJ), Brasil. E-mail: mcristina.ufrj@gmail.com

8 Enfermeira. Pós-graduanda. Universidade Federal do Rio de Janeiro (UFRJ), Brasil. E-mail: lianebruna@gmail.com

9 Professor. Universidade do Estado do Rio de Janeiro (UERJ), Brasil. E-mail: antoniedu@gmail.com

10 Professora. Universidade do Estado do Rio de Janeiro (UERJ), Brasil. E-mail: ravini_uerj@hotmail.com

11 Professor. Universidade Federal de Alfenas (UNIFAL - MG), Brasil. E-mail: alexandreotelles@gmail.com

12 Professora. Universidade Federal do Rio de Janeiro (UFRJ), Brasil. E-mail: halfoun@uol.com.br

13 Professora. Universidade Federal do Rio de Janeiro (UFRJ), Brasil. E-mail: mariakatia.gomes@gmail.com

Recebido em: 08/10/2020 | Aprovado em: 19/11/2020

DOI: 10.12957 /interag.2020.50780

\section{Resumo}

Relato de experiência de atividades extensionistas. Objetivou: Discutir os significados das vivências da equipe executora do projeto referentes à educação em saúde na modalidade lúdico-teatral, realizadas em escola de ensino fundamental do Município do Rio de Janeiro. Público-alvo: crianças de 6 a 12 anos. A abordagem qualitativa do tipo etnográfica apoiada em Geertz possibilitou a identificação de significados das experiências da equipe. Os significados das temáticas, apoiados no interacionismo simbólico, das ações de educação em saúde são culturalmente apreendidos desde a infância, sendo constantemente reelaborados e reproduzidos no decorrer da vida, porém mantendo padrão de certo modo medicalizante. A centralidade das ações estava representada por significados relacionados predominantemente ao modelo biomédico, mas no decorrer das ações distintas percepções emergiram. Ressalta-se o potencial de reflexão/crítica dos extensionistas sobre a realidade.

\begin{abstract}
Experience report of extension activities. Objective: Discuss the meanings of the experiences of the project's executing team regarding health education in the playful-theatrical modality, held at the elementary school in the city of Rio de Janeiro. Target audience: children aged 6 to 12 years. The qualitative approach of the ethnographic type supported by Geertz made it possible to identify meanings of the team's experiences. The meanings of the themes, supported by symbolic interactionism, of health education actions are culturally apprehended since childhood, being constantly reworked and reproduced throughout life, while maintaining a somewhat medicalizing pattern. The centrality of the actions was represented by meanings related predominantly to the biomedical model, but in the course of the actions, different perceptions emerged. The extensionists' potential for reflection / criticism about reality is emphasized.
\end{abstract}


Palavras-chave: Saúde Escolar; Promoção da

Saúde; Educação Popular; Atenção Primária à Saúde.
Keywords: School Health; Health Promotion; Popular

Education; Primary Health Care.

Área temática: Saúde.

Linha de extensão: Saúde Humana; Infância e Adolescência; Artes Cênicas (Teatro).

\section{Introdução}

O presente trabalho consiste num relato de experiência discente das atividades do projeto de extensão intitulado "Teatro em Saúde", com características de pesquisa voltadas para a Atenção Primária à Saúde (APS) no contexto do Programa Saúde na Escola (PSE). Desenvolvem-se ações de Educação em Saúde, junto à comunidade escolar da rede pública de ensino do Município do Rio de Janeiro. O projeto está credenciado na Faculdade de Medicina da UFRJ, integrando as atividades das unidades da Estratégia de Saúde da Família (ESF) nas quais são desenvolvidas atividades do Internato Integrado de Medicina de Família, Saúde Mental e Saúde Coletiva, e no PCI-AIS (Atenção Integral à Saúde). Entretanto, como um projeto de extensão está apoiado no pilar da interdisciplinaridade e interprofissionalidade, estando disponível aos discentes de todas as áreas, inclusive das ciências humanas, artes, ciências exatas, entre outras. Ademais também está receptivo à participação de discentes externos à UFRJ.

O ambiente escolar deve ser compreendido como espaço educacional dinâmico de movimento social que promove a intersetorialidade mediante a articulação de ações, envolvendo a saúde e segurança pública e tem como uma de suas finalidades a construção coletiva de ações e estratégias potencializadoras para redução das desigualdades sociais existentes ${ }^{1},,^{2}, 3$.

A experiência na implementação de ações de promoção da saúde/prevenção de danos aos escolares favoreceu na escola um ambiente de formação do cidadão com possibilidades de aprendizagem mútua, tanto para a equipe do projeto quanto para os escolares. Assim, foi possível a articulação entre os conteúdos e as práticas de educação 
em saúde numa perspectiva ampliada do cuidado. O teatro foi escolhido como estratégia pedagógica, pois possibilita alcançar ludicamente indivíduos, grupos e coletividades, facilitando a execução da ação e a reconstrução do conhecimento. $O$ teatro pode ser visto como um jogo dramático completo, pois consegue envolver a criança integralmente, abrangendo a criatividade e o aprendizado por meio da descontração; desta maneira propicia a troca de saberes ${ }^{4,5,6}$.

O referencial teórico utilizado está vinculado aos conceitos de cultura de Geertz. Dada a complexidade da temática propõe-se tecer uma breve consideração sobre o conceito abordado. Numa perspectiva semiótica, "o homem é um animal amarrado a teias de significados que ele mesmo teceu". A cultura deve ser entendida como sendo "essas teias e a sua análise, uma ciência interpretativa à procura do significado ${ }^{7 ”}$. A abordagem etnográfica visa compreender holisticamente os modos de vida dos grupos sociais, apoiada no interacionismo simbólico. Esta concepção "defende a ideia de que as pessoas agem a partir dos significados que elas atribuem a suas ações, às outras pessoas e aos objetos a partir da interação" ${ }^{8 ”}$

\section{Objetivos}

- Discutir os significados das vivências da equipe executora do projeto referentes à educação em saúde na modalidade lúdico-teatral, realizadas em escola de ensino fundamental do Município do Rio de Janeiro.

\section{Justificativa}

Para a universidade tem sido um desafio ampliar os cenários de ensino para os cursos de graduação na área da saúde, à luz das Diretrizes Curriculares Nacionais (DCN's - 2001 e 2014). O Sistema Único de Saúde (SUS) preconiza que o ensino de graduação deve acontecer a partir do conhecimento da realidade social e das necessidades de saúde da população brasileira, incluindo os direitos das crianças e a sua inserção plena na cidadania ativa. Isto se constitui como condição fundamental para a formação de 
profissionais de saúde enquanto agentes de transformação. Esta experiência permitiu à equipe entender o universo das crianças, nas suas contradições, complexidade e potencialidades ${ }^{9}$.

\section{Metodologia}

Trata-se de um relato de experiência com abordagem qualitativa, a respeito das atividades de um projeto de extensão, que visa à produção de conhecimento sobre a promoção da saúde na escola e ao fortalecimento da interação dialógica e aliança de saberes entre a equipe e os sujeitos envolvidos. O propósito foi favorecer o protagonismo e participação popular nas questões que envolvem a temática de saúde em uma instituição de ensino fundamental. Como estratégia educacional foi utilizado o teatro na modalidade musical como intervenção educativa. Este relato é produto da observação, registro e análise das atividades desenvolvidas. Segundo $\mathrm{MINAYO}^{10}$ as concepções qualitativas são: "aquelas capazes de incorporar a questão do SIGNIFICADO e da INTENCIONALIDADE como inerentes aos atos, às relações, e as estruturas sociais, sendo essas últimas tomadas tanto no seu advento quanto na sua transformação, como construções humanas significativas”.

O enfoque etnográfico foi empregado na busca da compreensão do universo de significados, valores e atitudes da equipe no contexto social observado 9 . Essa abordagem tem sido amplamente utilizada com sucesso em muitas áreas do conhecimento, inclusive no campo da saúde, tanto em pesquisas como nas atividades de extensão universitária. A opção pela etnografia deveu-se pela possibilidade de compreender o contexto escolar, a partir da realidade da comunidade atendida pelo projeto. Buscou-se entender a forma de vida do grupo social, pautada no interacionismo simbólico como marco conceitual ${ }^{8}$. Para tanto a equipe executora realizou uma imersão no cenário de desenvolvimento das ações educativas. Isto permitiu uma aproximação da equipe do projeto com a comunidade escolar favorecendo a adoção de estratégias para a criação de vínculo, bem como permitindo a troca e a aliança de saberes. 
Os dados concernentes a esse relato de experiência foram obtidos a partir da captura das narrativas oriundas dos diários de campo da equipe. Ao escolher a escola como cenário, partiu-se da premissa que neste espaço social, a cultura local e os diferentes saberes se entrecruzam, favorecendo a criação e recriação de formas de pensar, agir e compreender o mundo. O pensar pode ser definido como um tráfego de símbolos significantes tais como palavras, gestos, sons, desenhos, dentre outros elementos que podem ser utilizados para imprimir um significado à experiência?

Para inserção da equipe no cenário, o projeto foi apresentado à direção e professores. Foram realizadas reuniões com os pais e com as crianças para explicar os objetivos do projeto. Aos pais, foi solicitada autorização formal mediante assinatura de termo específico para que seus filhos pudessem participar das atividades extensionistas. Com o intuito de valorizar o protagonismo infantil foi coletado o assentimento das mesmas. A participação foi voluntária e sem vantagens financeiras. Buscou-se identificar as lacunas de conhecimento referentes às temáticas de promoção da saúde, com vistas à problematização e potencialização do ensino da educação em saúde na graduação. Os temas foram escolhidos pela comunidade escolar: Bullying, violência doméstica e urbana, reciclagem e sustentabilidade ambiental, arboviroses, importância da higiene corporal e da alimentação saudável, prevenção de acidentes na infância, entre outros.

\section{Descrição do Cenário Extensionista e Público-Alvo}

As atividades ocorreram em uma escola filantrópica de ensino fundamental e médio situada no Centro da Cidade do Rio de Janeiro, vinculadas à clínica de família da Coordenadora de Saúde da Área de Planejamento (CAP 1.0). Foram atendidas as crianças em idade escolar (6 a 12 anos). A instituição ocupa um espaço de $15.000 \mathrm{~m}^{2}$ e contém 101 funcionários. O corpo docente dispõe de 15 professores possuindo atualmente 347 alunos. O horário de funcionamento da educação infantil é diurno. A cantina está aberta de $11 \mathrm{~h} 30 \mathrm{~min}$ até às 14 horas e a biblioteca em período integral. 


\section{Relato de Experiência / Discussão}

Para o desenvolvimento das atividades considerou-se as ações educativas/ dramatizações fundamentais para a promoção da participação ativa da comunidade escolar, o que possibilitou uma aproximação da equipe com todos que estavam implicados no processo. Sob este prisma o referido projeto adotou a educação popular em saúde como ferramenta de intervenção propiciando condições essenciais para o exercício de cidadania. Sendo assim, foram aplicadas metodologias ativas na educação em saúde para o incremento da participação social, e protagonismo das crianças, numa perspectiva compreensiva das dimensões crítica e ativa desse processo. Dessa maneira as atividades realizadas atenderam aos eixos estratégicos da Política Nacional de Educação Popular em Saúde, principalmente quanto à participação popular, cuidado em saúde, diálogos multiculturais e produção de conhecimento ${ }^{11}$.

A imersão da equipe no cenário escolar permitiu a aplicação de alguns atributos essenciais e derivativos da APS, tais como: a) criação de vínculo e cuidado longitudinal, uma vez que a equipe desenvolvia suas atividades de forma regular e contínua, otimizando as ações do PSE por promover uma interface entre a Universidade e a Clínica da Família, fazendo que as ações não sejam fragmentadas; b) integralidade, ao adotar estratégias de educação em saúde que vislumbraram a criança de uma forma holística com necessidades biopsicossociais; e c) abordagem familiar e competência cultural, já que o teatro como ferramenta facilitadora da educação popular em saúde, consegue interligar esses conceitos, inserindo no contexto da promoção da saúde o papel da família no processo educativo $^{3,12}$.

Ademais as atividades desenvolvidas pelo projeto auxiliam na formação de recursos humanos na assistência em saúde, à luz das DCN's, por permitir ao estudante aprimorar habilidades de comunicação com o usuário, descentralizando o processo de cuidado da figura profissional para tornar a comunidade protagonista do seu próprio processo de educação em saúde ${ }^{13,14}$. 
Figura 1: Distribuição dos estudantes extensionistas participantes do projeto "Teatro em Saúde”, segundo idade e cor/raça. Fonte: Própria.

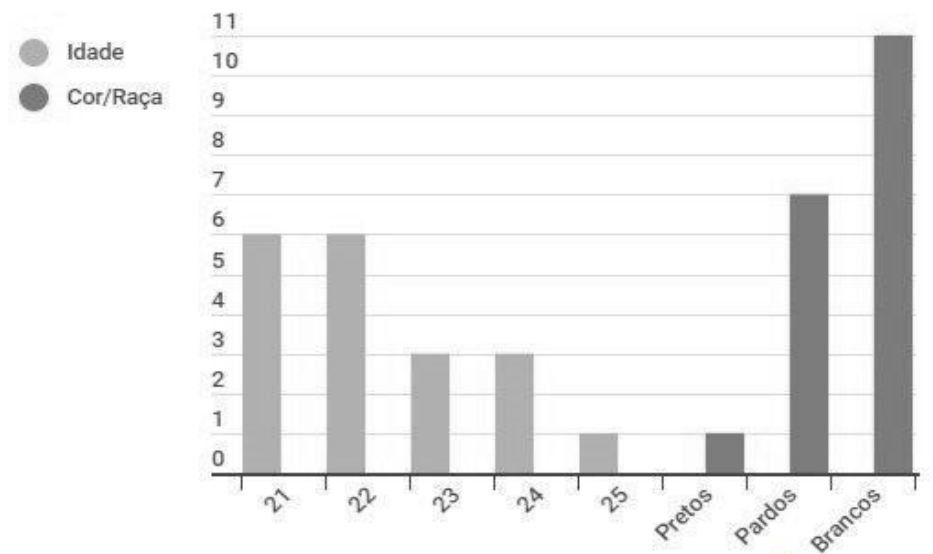

Os dados da figura 1 apontam que dos 18 graduandos que compuseram a equipe do projeto, a maioria $(63,16 \% / \mathrm{n}=12)$ se concentra na faixa etária entre 21 e 22 anos. Em relação à cor/raça autodeclarada pelos extensionistas, a maioria se identificou como branco $(57,89 \% / \mathrm{n}=11)$.

Figura 2: Distribuição dos estudantes extensionistas participantes do projeto "Teatro em Saúde”, segundo período e gênero. Fonte: própria.

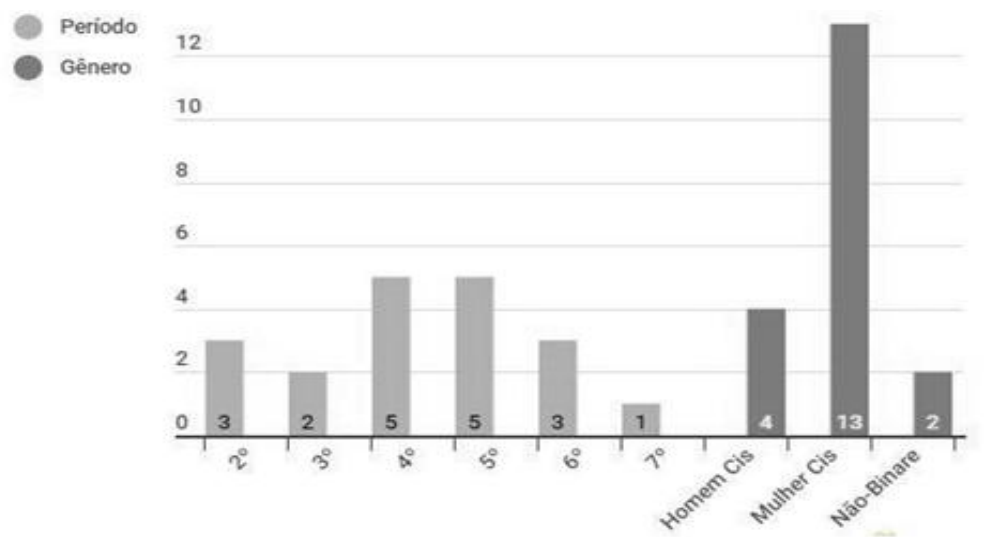


Os dados da figura 2 mostram que a maioria $(52,63 \% / n=10)$ cursava entre o quarto e quinto período. Desses alunos 17 cursavam enfermagem na UFRJ e apenas 1 cursava Design Gráfico na Universidade Estácio de Sá. Quanto ao gênero, a maioria dos extensionistas $(68,42 \% / \mathrm{n}=13)$ se autodeclaram mulher cis. No que tange à nossa vivência pudemos observar o predomínio de ações pautadas no modelo biomédico, higienista, assistencialista e normativo, em detrimento dos componentes que privilegiam o contexto da população, seus modos de vida, bem como os determinantes de saúde da comunidade escolar ${ }^{15,16}$. Por outro lado, as atividades do projeto foram profícuas, pois favoreceram o protagonismo infantil na comunidade escolar, considerando o privilégio da fala das crianças estimulando a participação em todas as etapas de elaboração das ações educativas. Isto permitiu uma aproximação dialógica durante todo o processo de realização das ações educativas. Percebeu-se que muitos escolares interagiam com a equipe trocando informações sobre autocuidado, tomando como base características peculiares das personagens das peças. No tocante às crianças, verificamos o interesse genuíno em participar do projeto. Em que pese o fato de que as mesmas já apresentavam um conhecimento prévio acerca de diversas temáticas relativas às práticas de promoção da saúde. Entretanto, o cerne dessas informações englobava, geralmente, conteúdos numa perspectiva higienista, com destaque para as ações de cuidado corporal, tais como higiene, alimentação e práticas de exercícios físicos. As experiências da equipe com as crianças evidenciaram que as temáticas emergentes captaram muito mais atenção das crianças, tais como: violência e bullying, impacto do meio ambiente na saúde, prevenção de acidentes na infância e cidadania. Foi gratificante identificar, mesmo que de forma tímida, sinais que indicam os pródromos das mudanças de postura em algumas crianças. Observou-se que alguns escolares, geralmente os maiores ( 9 a 12 anos de idade) se posicionaram, criticamente em relação ao paradigma prescritivo e de reforço negativo/proibitivo. Isto nos permitiu encorajá-las a adotarem uma abordagem mais realista ao contexto de suas próprias vidas. Segue abaixo a teia de significação das práticas educativas que permearam a percepção da equipe do projeto antes da realização das peças teatrais. 
Figura 3: Teia de Significados das Experiências da Equipe Executora sobre as Ações Educativas. Fonte: própria.

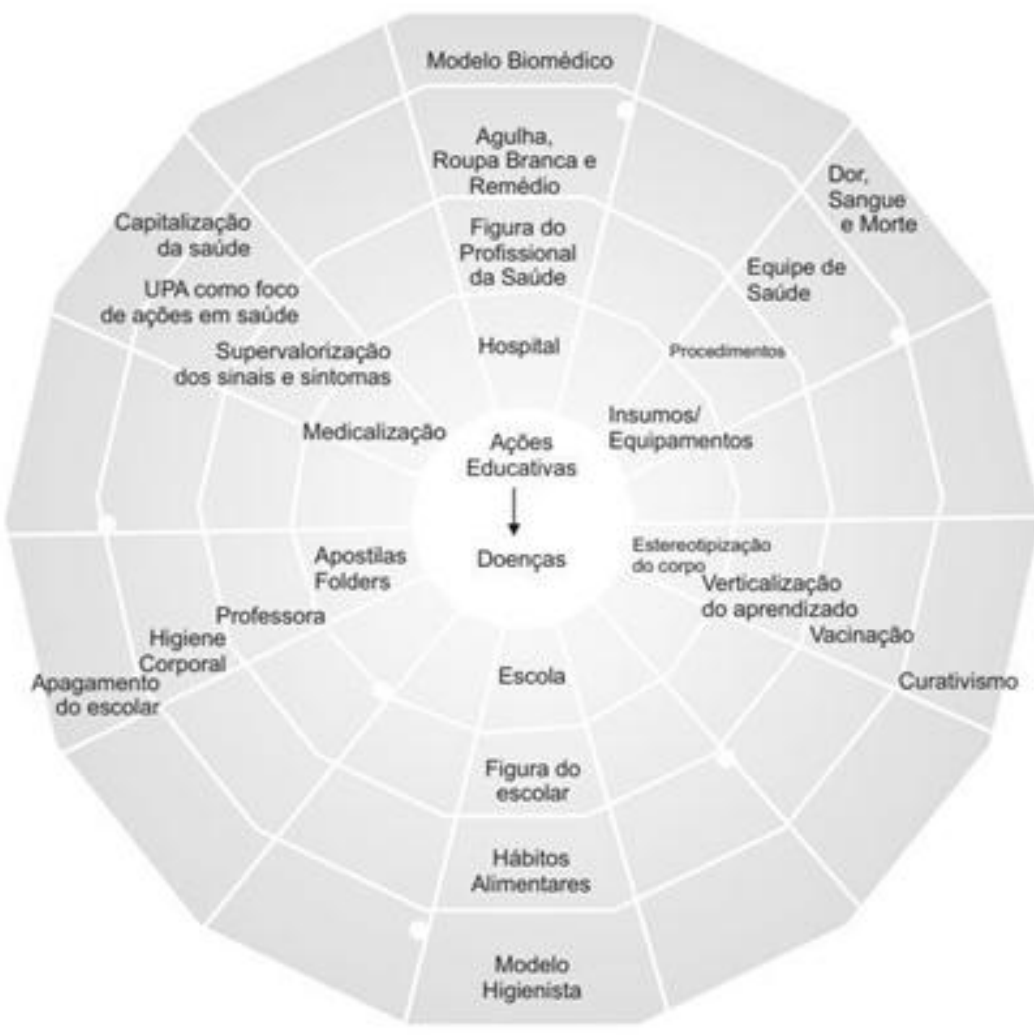

A partir da leitura da teia observa-se o atual modelo hegemônico de saúde que influencia decisivamente a operacionalização das ações educativas no cenário da escola. Ademais a teia evidencia o processo dinâmico que ocorre com o sistema de códigos culturais dos participantes. A construção da teia ocorreu a partir das percepções prévias da equipe sobre as práticas de promoção da saúde. Em qualquer lugar da teia, em cujo centro estão as ações educativas focadas na doença, os significados estão entrelaçados influenciando e sendo influenciados mutuamente, mantendo o padrão cultural que estrutura as práticas de saúde na escola e repercute de diferentes maneiras no cuidado dos escolares. A dinâmica existente entre os diversos significados atribuídos pela equipe é que, por exemplo, a partir do termo "doença" a mesma pode ser entendida como ligada 
inicialmente ao lugar onde é "tratada" ("Hospital") e aos agentes que dela cuidam ("Figura do profissional de saúde"). Esta percepção veio à tona por ocasião da preparação da equipe para inserção no cenário. Nessas rodas de conversa preparatórias percebeu-se a reprodução de clichês da visão biomédica e higienista. Isto porque o modelo flexneriano tem presença muito marcante nos cursos de graduação em saúde.

Entretanto, após sua imersão, conforme a equipe executora se apropriou das atividades do projeto, ocorreu uma ressignificação acerca das temáticas no sentido de ampliar a visão dos estudantes para que fosse abarcado o paradigma da integralidade. Isto permitiu à equipe avançar na reflexão à respeito da influência das relações interpessoais, afetividade, meio ambiente, suporte social, dentre outros, componentes do processo saúde-doença. Cabe destacar que a ressignificação das práticas educativas elaboradas pela equipe, após a realização das peças teatrais, será tema de um artigo a ser publicado posteriormente. Outros pontos da teia são afetados por essa mesma linha de significação quando, por exemplo, o local e os profissionais de saúde são correlacionados ao tratamento ("Dor, sangue e morte") ou ao conjunto de orientações para o cuidado de sua saúde ("estereotipização do corpo"). Pode-se inferir que os significados referentes às temáticas de educação em saúde são culturalmente apreendidos desde a infância, sendo constantemente reelaborados e reproduzidos durante toda a vida, porém mantendo certo padrão medicalizante, no qual se verifica o apagamento do sujeito, a supervalorização da tecnologia, a centralidade das ações focados no controle de doenças e normatização do corpo, a priorização de práticas higienistas e de abordagens curativas em detrimento das ações de promoção da saúde ${ }^{1}$. A vivência da equipe no cenário de prática evidenciou o papel da família, da comunidade, dos sentimentos e interações humanas e dos serviços de saúde, para o bem-estar psicossocial da comunidade escolar.

\section{Considerações Finais}

A educação popular foi o ponto de partida para a compreensão da realidade da comunidade escolar. O teatro foi utilizado como ferramenta estratégica de diálogo que 
permitiu a imersão da equipe no universo infantil, favorecendo a aproximação da criança com os temas abordados além de fomentar a transformação social e o desenvolvimento humano por meio da arte de todos os envolvidos.

Podem-se elencar os benefícios e interações do projeto para com os diferentes sujeitos envolvidos nas atividades realizadas. Para a escola, observa-se subsídio na incorporação das temáticas referentes à saúde no cotidiano escolar, as quais fazem parte das Diretrizes e Bases da Educação Brasileira. Além disso, a escola esteve altamente receptiva às atividades do projeto, destacando-se o apoio de infraestrutura mediante a cessão do espaço físico, recursos materiais e humanos, divulgação das atividades extensionistas juntamente aos pais/responsáveis. Para o escolar, destaca-se o protagonismo das crianças na condição de usuárias do SUS e multiplicadoras das ações de promoção da saúde.

Para os acadêmicos participantes no projeto, pode-se inferir a contribuição das atividades de extensão na ressignificação das práticas educativas em saúde, favorecendo que sua formação profissional tenha como base a centralização do cuidado no sujeito, a valorização das necessidades biopsicossociais e o diálogo da comunidade, promoção de um ambiente que permita aliança e a troca entre os saberes acadêmico e popular, além de outras estratégias que facilitam a criação de vínculo com o individuo, família e comunidade para uma melhor assistência em saúde. Destaca-se que, os graduandos ao atuar na atenção primária à saúde devem utilizar-se desta ferramenta para auxiliar na prevenção de danos e construção de saberes sobre o autocuidado em saúde.

\section{Referências}

1. LUCAS, Eduardo Alexander Júlio César Fonseca. Os significados das práticas de promoção da saúde na infância: um estudo do cotidiano escolar pelo desenho infantil. 2013. Tese (Doutorado em Saúde Materno Infantil) - Faculdade de Saúde Pública, Universidade de São Paulo, São Paulo, 2013. Disponível em: <http://www.teses.usp.br/teses/disponiveis/6/6136/tde-07052013-163232/>. Acesso 
em: 10 nov. 2019.

2. BRASIL. Ministério da Saúde. Secretaria de Atenção à Saúde. Departamento de Atenção Básica. Saúde na escola. Brasília: Ministério da Saúde, 2009. 96 p. il. (Série B. Textos Básicos de Saúde. Cadernos de Atenção Básica, n. 24).

3. BRASIL. Ministério da Saúde. Secretaria de Atenção à Saúde. Departamento de Atenção Básica. Passo a passo PSE (Programa Saúde na Escola) - Tecendo caminhos da intersetorialidade. Brasília: Ministério da Saúde, 2011.

4. NAZIMA, Tue Jolo; CODO, Carla Regina Bianchi; PAES, Irani Aparecida Dalla Costa; BASSINELLO, Greicelene Aparecida Hespanhol. Orientação em saúde por meio do teatro: relato de experiência. Rev Gaúcha Enferm., Porto Alegre (RS) 2008 mar; 29(1):147-5.

5. SARMENTO, Manuel Jacinto. As culturas da infância nas encruzilhadas da 2a modernidade. Braga: Instituto de Estudos da Criança, Universidade do Minho, 2003.

6. ARCOVERDE, S. L. A importância do teatro na formação da criança. In: Congresso Educação, Profissionalização Docente e Formação. Pontifícia Universidade Católica do Paraná, 2008. Anais. Disponível em: <https://educere.bruc.com.br/arquivo/pdf2008/629_639.pdf> Acessado em: 13 de Junho de 2016.

7. Geertz, Clifford. A Interpretação das Culturas. 1 1a ed. [Reimpr.], Rio de Janeiro: LTC, 2011.

8. FONTES, Rejane de Souza. Ensino colaborativo: uma proposta de educação inclusiva. Araraquara, SP: Junqueira \& Marin, 2009.

9. SANTOS, Alinne Neyane; SANTOS, Alice Nayara. O teatro e suas contribuições para educação infantil na escola pública. In: XVI ENCONTRO NACIONAL DE DIDÁTICA 
E PRÁTICAS DE ENSINO. Campinas, 2012. Disponível em: < http://www2.fct.unesp.br/docentes/geo/raul/geografia_humana2017/teatro_educa\%E7 \%E3o2.pdf>. Acessado em: 11 de Nov de 2019.

10. MINAYO, Maria Cecília de Souza. O desafio do conhecimento: pesquisa qualitativa em saúde. 8a Ed. São Paulo / Rio de Janeiro: Hucitec, 2004.

11. BRASIL. Ministério da saúde. Portaria № 2.761, de 19 de novembro de 2013. Institui a Política Nacional de Educação Popular em Saúde no âmbito do Sistema Único de Saúde (PNEPS-SUS). Brasília: Ministério da Saúde, 2013.

12. OliveIRA, Maria Amélia de Campos; PEREIRA, Iara Cristina. Atributos essenciais da Atenção Primária e a Estratégia Saúde da Família. Rev. bras. enferm. 2013. Disponível em:

<https://www.scielo.br/scielo.php?script=sci_arttext\&pid=S0034-71672013000700020> Acesso em: 28 Set 2020

13. BRASIL. Ministério da Educação. Parecer CNE/CES no 1.133/2001. Diretrizes Curriculares Nacionais dos Cursos de Graduação em Enfermagem, Medicina e Nutrição. Brasília: Ministério da Educação, 2001.

14. BRASIL. Ministério da Educação. Resolução No 3, de 20 de junho de 2014. Institui Diretrizes Curriculares Nacionais do Curso de Graduação em Medicina e dá outras providências. Brasília: Ministério da Educação, 2014.

15. BRASIL. Ministério da Saúde. Secretaria de Vigilância em Saúde. Política Nacional de Promoção da Saúde: Portaria no 687 MS/GM, de 30/3/2006. Brasília: Ministério da Saúde, 2006.

16. VALADÃO, Marina. Marcos. Saúde na Escola: um campo em busca de espaço na agenda intersetorial. 2004. Tese (Doutorado em Saúde Pública). Faculdade de Saúde Pública, Universidade de São Paulo, São Paulo. 